\title{
Answer to the Letter to the Editor of Prof. H.V. Crock concerning "A less invasive surgical approach in the lumbar lateral recess stenosis: direct approach to the medial wall of the pedicle" (Colak A et al (2008) Eur Spine J 17:1745-1751)
}

\author{
Hakan Şimşek • Ahmet Çolak
}

Published online: 8 April 2009

(C) Springer-Verlag 2009

\section{To the Editor}

We have read the above letter and evaluated it in view of the references.

Though no satisfactory guideline has been established as to determine the efficacy of the decompression in the lateral recess stenosis, we advised, in our paper that a thorough decompression should consist loosening the entry and exit zones, and delineating the medial wall of the relevant pedicle lateral to the root. In such stenotic canals, the root may be very sensitive and any contact with the afflicted root may yield contraction of the limb. However, this response is very much bound to the level of anesthesia to the patient. Deep anesthesia is concurrent with no response, and may not allow contraction of the relevant limb. In our surgical procedure, we tried to abstain from contact with the root of the operated level. Although we also sometimes observed rapid refilling of the perineural veins of the affected root, we have been considering that absence of refilling of the veins did not always mean ineffective and insufficient decompression. But our colleague's current study, in fact, will be a useful and instructive complement.

Thank you for your interest and contributions. 